\section{Cadastro ampliado em saúde da família como instrumento gerencial para diagnóstico de condições de vida e saúde}

\author{
The expanded enrolment form in the Brazilian \\ Family Health Program as a management tool \\ for diagnosis of living and health conditions
}

\author{
Arnaldo Sala 1 \\ Oziris Simões 1 \\ Carla Gianna Luppi 2 \\ Miryan Cristina Mazziero 2
}

\footnotetext{
1 Faculdade de Medicina, Universidade de São Paulo, São Paulo, Brasil.

2 Faculdade de Ciências

Médicas, Santa Casa de São Paulo, São Paulo, Brasil.

Correspondência A. Sala

Centro de Saúde Escola Prof. Samuel B. Pessoa, Faculdade de Medicina Universidade de São Paulo. Av. Vital Brasil 1490, São Paulo, SP 05503-000, Brasil. arnasala@usp.br
}

\begin{abstract}
As a consequence of the introduction of the Family Health Program in the city of São Paulo, Brazil, a pilot experiment was conducted with an expanded enrolment form for gathering information on families at two school health services. The aim of the study was to analyze this enrolment form as a management tool capable of identifying differences and inequities in each area. The collected data provided the basis for generating six related indicators: time of residence in the area, family members per bedroom, per capita family income, number of children as a percentage of total family members, schooling, and health insurance coverage. A compound indicator was constructed, called the Mean Living Standard Score, in addition to another indicator - Mean Score/ Basic Health Care Information System - from the three indicators existing in form A of the Basic Health Care Information System. The results identified contiguous geographic areas with different living standards. The two scores showed similar discriminatory power. In conclusion, it is possible to differentiate and discriminate sub-areas, thereby highlighting the need to organize different health actions for each sub-area.
\end{abstract}

Health Family Program; Information Systems; Management

\section{Introdução}

O gerenciamento da Unidade Básica de Saúde (UBS) tem tradicionalmente, no Brasil, adquirido dimensão que transcende a questão da administração dos recursos humanos e materiais e se configura como uma atividade que compõe, além da administração propriamente dita dos recursos, a atribuição de organizar as atividades a fim de implementar uma política de saúde em que o que está em jogo são elementos tais como acesso e satisfação da população que demanda serviços de saúde, controle de doenças em uma população, vigilância epidemiológica, promoção da saúde e prevenção de agravos, controle de meio ambiente, entre outras.

Para realizar essas atividades, a gerência da UBS lança mão de um conjunto de técnicas relacionadas à medicina, à epidemiologia, à educação, ao controle do ambiente entre outras que, em conjunto articulado, poderiam ser tratadas como uma Técnica Sanitária 1. Esta Técnica Sanitária teria como elementos nucleares: (1) a população com seus processos de saúde e adoecimento; (2) a oferta de ações em saúde que incide diretamente sobre o coletivo; (3) a oferta de ações em saúde que incide imediatamente sobre os indivíduos, mas que possuem impacto sobre o coletivo.

A informação, para esta gerência que articula este tipo de técnica, ganha o sentido de 
possibilidade de apreensão de processos de vida e adoecimento de populações, bem como de acompanhamento das ações e avaliação de seus resultados (impacto epidemiológico, relação custo-efetividade, satisfação etc.).

O Programa Saúde da Família (PSF), concebido como estratégia para a organização da atenção básica no SUS, tendo como princípios gerais a universalidade, a integralidade, o enfoque na prevenção, enfoque na família, população adstrita, participação comunitária e controle social 2, pode ter o seu gerenciamento local entendido como orientado por esta Técnica Sanitária. Entre as múltiplas necessidades de informação aí presentes destaca-se, neste estudo, a informação acerca da população adstrita, como requisito básico para a adequada organização das ações.

Para o PSF, a família aparece como a unidade de ação programática, e não apenas o indivíduo 3,4 Deste modo, a família deve ser tomada como unidade de análise, pois é o objeto privilegiado de intervenção.

Considera-se que os indivíduos são definidos, agora, com base em sua relação na família. Como resultado de "um sistema de interação interdependente, a família mantém seus membros implicados na capacidade de alterar o comportamento dos outros com seus próprios movimentos" 5 (p. 431). Os autores destacam, ainda, a noção de "família como um sujeito" que é distinto dos indivíduos que a compõem. Consideram a família como um contexto que pode constituir-se como elemento protetor ou fragilizador dos seus membros 5 .

Do mesmo modo como a organização familiar interage com os indivíduos, um conjunto de famílias que se inter-relacionam, constituindo uma comunidade, também pode modificar o desempenho de cada família, tornandose esta comunidade elemento protetor ou fragilizador de cada família.

Esta última consideração é particularmente importante no contexto da saúde da família, em que o trabalho não se esgota na ação sobre cada família, mas compreende também a dinâmica da comunidade ou de um conjunto social. Paim 6 aponta para a noção de que a exposição aos riscos à saúde decorrem da posição ocupada pelos indivíduos no espaço social e que, por outro lado, grupos de indivíduos serão normativos em relação ao meio, na dependência de uma dimensão material da vida e de uma dimensão cultural e simbólica. O espaço geográfico é construído socialmente, sendo as di- ferenças verificadas no primeiro resultado das relações presentes no segundo.

A questão da espacialização das condições de vida e adoecimento de uma população é especialmente relevante na implementação do PSF, sendo oportuno reter o conceito de território-processo, "como um espaço em permanente construção, produto de uma dinâmica social em que se tensionam sujeitos sociais postos na arena política" 7 (p. 166).

Uma área geográfica adstrita à unidade de saúde da família geralmente é tomada como possuindo características bastante homogêneas, por tratar-se de uma parte de um bairro próximo à unidade de saúde. Este território, além de constituir-se em espaço alvo de atuação da equipe, conforma uma certa identidade comunitária a este grupo de famílias, uma vez que possui situação sócio-cultural com razoável homogeneidade e, freqüentemente, algum tipo de atividade associativa.

Deste modo, não seria surpresa verificar que a organização do trabalho dirigido a esta comunidade a avalie operacionalmente como homogênea em sua condição de vida e de saúde, estabelecendo, assim, rotinas de trabalho na unidade de saúde e nos domicílios adequadas a um padrão médio de vida e de adoecimento estimados pela equipe de saúde. As variações efetivamente encontradas entre os indivíduos e as famílias seriam tratadas no âmbito individual, sem a necessidade de uma recomposição na organização do trabalho que pudesse "discriminar positivamente" aqueles indivíduos ou famílias que fogem do padrão médio de vida e saúde da comunidade.

No entanto, parece pertinente e oportuno negar esta hipótese da homogeneidade da situação sócio-cultural, econômica e de situação de saúde desta comunidade adstrita a uma unidade de saúde da família, em razão do reconhecimento da óbvia heterogeneidade oculta por trás de indicadores que espelham a situação média de vida e saúde. Mendes et al. 7 chamam a atenção para o fato de que os problemas de saúde não se distribuem de forma simétrica no espaço circunscrito por um serviço de saúde; para os autores, o "Território-microárea” seria o espaço relativamente homogêneo sobre o qual seria possível exercer "operações de discriminação positiva”.

Nestes termos, ao reconhecer diferenças na situação de vida e saúde destas microáreas e famílias, em que estão embutidos os fatores determinantes do adoecimento e das possibili- 
dades de efetivo acesso ao cuidado em saúde, abrem-se possibilidades mais efetivas de atuação da unidade de saúde.

Já é amplamente reconhecida a necessidade de discriminar, no espaço urbano de uma cidade, os diferentes gradientes de riqueza/pobreza (qualidade de vida) a que grupos de população estão expostos, uma vez que estas diferentes situações são geradoras de diferentes perfis de adoecimento. Akerman 8 mostra a relação entre um indicador composto por "renda familiar per capita", "percentual de pessoas analfabetas e com primário incompleto", "percentual de residências ligadas à rede de esgoto", "consumo per capita de água" e "número de pessoas por domicílio" e os distintos perfis de mortalidade em diferentes distrito e subdistritos na cidade de São Paulo, Brasil, agrupados por este indicador em quatro zonas homogêneas. Por sua vez, Barros 9 apresenta perfis de mortalidade para a cidade de Campinas, São Paulo, apontando para o fato de que a desigualdade em saúde, tomada por indicadores de mortalidade, esteja referida a um espaço sócio-geográfico definido.

Ao apreender distintas dinâmicas sócio-vitais, sejam estas reconhecidas valendo-se do estudo dos indivíduos, das famílias ou de relações comunitárias presentes no território, fica posta a pré-condição para orientar e adequar a organização do trabalho com intuito de uma discriminação positiva de indivíduos e grupos potencialmente mais expostos aos agravos à saúde e com maior dificuldade de acesso a ações em saúde, seja no âmbito do cuidado individual, seja no âmbito da família, seja no âmbito de ações que incidam diretamente sobre o território.

É com este tipo de preocupação que foi realizada uma experiência piloto de cadastramento de famílias adstritas a unidades de saúde no Município de São Paulo, a partir do final do ano de 2001. Esta experiência se originou fundamentada em duas ordens de preocupações: a primeira, no sentido de ampliar a coleta de dados incluindo questões que seriam relevantes para o contexto de um grande centro urbano, o que permitiria um melhor conhecimento das famílias da área adstrita, seja para ação imediata de cuidado em saúde para estas famílias, seja para orientar a organização gerencial do trabalho na unidade de saúde; a segunda, no sentido de aproveitar a oportunidade do cadastramento das famílias para coletar dados requisitados pelo Cartão Nacional de Saúde, cuja implantação estava se iniciando no município.

O objetivo deste trabalho é um estudo exploratório do cadastro ampliado enquanto ins- trumento para diagnóstico de saúde de uma população abrangida pelo PSF. Tem como perspectiva a possibilidade de discriminar diferenças e iniqüidades presentes em cada área adstrita. Como objetivos secundários, aparece a comparação entre os indicadores construídos com base no cadastro com a impressão mais subjetiva dos agentes comunitários de saúde (ACS) acerca das suas microáreas, e a comparação entre as possibilidades de diagnóstico de saúde proporcionado pelo cadastro ampliado, comparando com as possibilidades analíticas disponíveis nos dados da Ficha A do Sistema de Informação da Atenção Básica (SIAB).

\section{Metodologia}

Um cadastro ampliado foi elaborado, contendo os dados da Ficha A do SIAB, os dados requisitados pelo Cartão Nacional de Saúde, alguns novos agravos à saúde não presentes na Ficha $\mathrm{A}$, situações de riscos à saúde e situações de violência.

O formato deste cadastro ampliado definiu uma primeira seção destinada à coleta de dados acerca do domicílio (características físicas) e de questões relacionadas aos temas da saúde que poderiam ter repercussões sobre o agrupamento familiar como um todo, tais como acesso a serviços de saúde, situações referidas de riscos à saúde, percepção de situações de violência, entre outras. Uma segunda seção foi destinada aos dados individualizados de cada um dos membros da família, considerando desde a identificação pessoal, dados vitais, escolaridade, ocupação, agravos e condições de saúde, situação vacinal e uso da unidade de saúde.

O cadastro foi implantado em 2002 em quatro equipes de PSF em dois Centros de Saúde Escola (CSE) na cidade de São Paulo, um deles localizado em área central da cidade, na região da Barra Funda (compreendendo bairro de ocupação mais antiga, áreas com predomínio de cortiços e área de condomínio mais recente), e outro em área periférica, na região do $\mathrm{Bu}$ tantã (compreendendo área exclusivamente de favela, nas vizinhanças do campus universitário da Universidade de São Paulo). Um programa especificamente desenvolvido para estas unidades gerencia a digitação e a emissão de relatórios informatizados.

Os dados do cadastro ampliado dos CSE da região da Barra Funda e do Butantã foram dispostos em dois arquivos de dados em formato $D B F$ : um deles referente às pessoas; já o outro, aos atributos de família e de domicílio. Estes 
dados refletem a posição do cadastramento das famílias no mês de julho de 2003.

Para a análise e elaboração do diagnóstico da população adstrita a cada uma das unidades de saúde, foram levadas em conta: (1) as variáveis pessoais relativas a agravos e condições de saúde referidos (problema mental, deficiência física, tabagismo, etilismo, epilepsia, hipertensão, diabete, tuberculose, bronquite, infecção respiratória aguda, antecedentes de internação), idade e escolaridade; (2) as variáveis tomadas como atributos da família, como renda familiar, número de pessoas no domicílio, número de cômodos do domicílio, cobertura de convênio de saúde e tempo de moradia da família no bairro. Este conjunto de atributos de indivíduos e de famílias foi agregado para cada microárea definida na implantação do PSF em cada uma das unidades de saúde consideradas.

Pautados nestas variáveis, foram construídos seis indicadores de situação de vida, cujas médias de seus valores foram calculadas para cada uma das microáreas. A escolha destes seis indicadores se fez pelo uso de critérios múltiplos, incluindo desde a referência da literatura (em que renda e escolaridade são indicadores já consagrados), respostas diversificadas (com o objetivo de detectar desigualdades) e facilidade na obtenção do dado (precisão). Os seis indicadores são: (1) tempo de moradia da família no bairro (maior tempo de moradia é considerado como indicador mais favorável); (2) número de pessoas por cômodo utilizado para dormir (menor número é considerado como indicador mais favorável); (3) renda familiar per capita, em salário-mínimo (maior renda familiar é considerado como indicador mais favorável); (4) percentagem de crianças com idade menor que 12 anos (menor percentagem de crianças é considerado como indicador mais favorável); (5) escolaridade (anos de estudo) entre 20 e 29 anos de idade (maior escolaridade é considerado como indicador mais favorável); (6) percentagem de famílias com cobertura de convênio de saúde (maior cobertura de convênio é considerado como indicador mais favorável).

Para a determinação de um valor sintético que pudesse expressar o conjunto dos indicadores, foi construído um escore médio de situação de vida (EMSV) de cada microárea valendo-se dos escores derivados dos valores dos seis indicadores, nos seguintes passos: os valores de cada um destes indicadores foi normalizado em escore com valores entre 0 e 1 , correspondendo ao valor 0 o escore mais favorável daquele indicador, e ao valor 1 , ao escore mais desfavorável; os números intermediários foram interpolados entre 0 e 1 . Por fim, foi calcu- lado o escore médio dos seis indicadores, para cada microárea - o EMSV.

De modo similar, foi construído, ainda, um escore médio para o qual foram incluídos apenas indicadores possíveis de serem obtidos com os dados presentes na Ficha A do SIAB, aqui denominado de escore médio do SIAB (EM/SIAB), qual sejam: média de pessoas por cômodo, percentagem de crianças com idade inferior a 12 anos e percentagem de famílias com cobertura de convênio de saúde.

Por fim, foi solicitado aos ACS que hierarquizassem as microáreas que compõem a área de atuação do CSE de área periférica, segundo a dificuldade para realização de trabalho em saúde. Calculou-se o escore de cada microárea que sintetiza a opinião subjetiva destes ACS (escore de opinião dos ACS), em que o valor 0 corresponde à microárea mais favorável e o valor 1 à microárea com maior dificuldade para a realização do trabalho. Este último procedimento foi realizado apenas no CSE de área periférica, uma vez que os relatórios parciais do cadastramento das famílias não foram divulgados até o momento da coleta da opinião dos ACS. No outro CSE, a divulgação precoce dos dados invalidou esta etapa.

Por meio de análise de regressão linear (análise em Epi Info) foram estudadas: (1) a correlação entre o EMSV e o escore de opinião dos ACS; (2) a correlação entre o EMSV e o EM/SIAB; (3) a correlação entre o EMSV e a prevalência de agravos à saúde referidos.

\section{Resultados}

Escore médio de situação de vida (EMSV) e hierarquização entre microáreas identificação de subáreas

Com o objetivo de discriminar melhor as diferenças entre as microáreas, tomando simultaneamente diferentes indicadores relativos ao domicílio ou às pessoas, foram montados as Tabelas 1 e 2 (correspondentes às áreas do Butantã e da Barra Funda, respectivamente), que procuram expressar as diferenças entre as microáreas no sentido de sua vulnerabilidade a agravos, visto que expressam, ainda que de modo impreciso, situações de vida que constituem o cotidiano destas populações.

Notem-se as diferenças entre as duas áreas, não só nos coeficientes totais de cada indicador que compõe o EMSV, mas também nos valores encontrados em cada uma das microáreas. Calculando o EMSV em conjunto para as duas áreas, obteremos que nove das dez micro- 
Tabela 1

Valores dos indicadores de situação de vida, do escore médio de situação de vida (EMSV), do escore de opinião dos agentes comunitários de saúde (ACS) e escore médio do Sistema de Informação da Atenção Básica (EM/SIAB), para a área do Butantã, São Paulo, Brasil.

\begin{tabular}{|c|c|c|c|c|c|c|c|c|c|}
\hline \multirow[t]{2}{*}{ Microárea } & \multirow[b]{2}{*}{$\begin{array}{l}\text { Tempo de } \\
\text { moradia }\end{array}$} & \multirow[b]{2}{*}{$\begin{array}{l}\text { Média de } \\
\text { pessoas por } \\
\text { cômodo }\end{array}$} & \multicolumn{3}{|c|}{ Indicadores de situação de vida } & \multirow[b]{2}{*}{$\begin{array}{l}\text { Convênio de } \\
\text { saúde (\%) }\end{array}$} & \multirow[t]{2}{*}{ EMSV } & \multirow{2}{*}{$\begin{array}{l}\text { Opinião } \\
\text { dos ACS }\end{array}$} & \multirow[t]{2}{*}{$E M / S I A B$} \\
\hline & & & $\begin{array}{l}\text { Renda familiar } \\
\text { per capita }\end{array}$ & $\begin{array}{l}\text { Crianças } \\
<12 \text { anos (\%) }\end{array}$ & $\begin{array}{l}\text { Média de } \\
\text { escolaridade } \\
\text { entre 20-29 } \\
\text { anos }\end{array}$ & & & & \\
\hline $37-1$ & 7,1 & 3,0 & 0,6 & 37,0 & 5,8 & 1,9 & 0,9 & 1,0 & 0,9 \\
\hline $37-2$ & 14,7 & 2,5 & 1,0 & 27,4 & 7,3 & 20,7 & 0,2 & 0,2 & 0,1 \\
\hline $37-3$ & 6,8 & 2,4 & 0,7 & 31,4 & 7,0 & 2,6 & 0,6 & 0,8 & 0,5 \\
\hline $37-4$ & 12,5 & 2,7 & 0,9 & 29,6 & 6,6 & 17,0 & 0,4 & 0,5 & 0,3 \\
\hline $37-5$ & 11,7 & 2,8 & 0,5 & 38,1 & 6,2 & 1,9 & 0,8 & 1,0 & 0,9 \\
\hline $37-6$ & 14,8 & 2,3 & 1,0 & 32,1 & 7,3 & 18,0 & 0,2 & 0,3 & 0,2 \\
\hline $38-1$ & 12,6 & 2,5 & 1,0 & 28,5 & 6,4 & 18,0 & 0,3 & 0,2 & 0,2 \\
\hline $38-2$ & 9,3 & 2,9 & 0,7 & 33,9 & 5,7 & 7,7 & 0,7 & 0,8 & 0,7 \\
\hline $38-3$ & 13,6 & 2,5 & 1,3 & 26,9 & 8,0 & 20,9 & 0,1 & 0,0 & 0,1 \\
\hline $38-4$ & 11,6 & 2,3 & 1,1 & 28,8 & 7,2 & 20,6 & 0,2 & 0,3 & 0,1 \\
\hline $38-5$ & 10,5 & 3,2 & 0,5 & 37,4 & 5,6 & 3,9 & 0,9 & 0,6 & 0,9 \\
\hline $38-6$ & 12,4 & 2,6 & 0,9 & 27,8 & 7,3 & 13,1 & 0,3 & 0,8 & 0,3 \\
\hline Butantã & 11,6 & 2,7 & 0,9 & 31,3 & 6,7 & 13,0 & - & - & - \\
\hline
\end{tabular}

Tabela 2

Valores dos indicadores de situação de vida, do escore médio de situação de vida (EMSV) e escore médio do Sistema de Informação da Atenção Básica (EM/SIAB), para a área da Barra Funda, São Paulo, Brasil.

\begin{tabular}{|c|c|c|c|c|c|c|c|c|}
\hline \multirow[t]{2}{*}{ Microárea } & \multirow[b]{2}{*}{$\begin{array}{l}\text { Tempo de } \\
\text { moradia }\end{array}$} & \multirow[b]{2}{*}{$\begin{array}{l}\text { Média de } \\
\text { pessoas por } \\
\text { cômodo }\end{array}$} & \multicolumn{3}{|c|}{ Indicadores de situação de vida } & \multirow[b]{2}{*}{$\begin{array}{l}\text { Convênio de } \\
\text { saúde (\%) }\end{array}$} & \multirow[t]{2}{*}{ EMSV } & \multirow[t]{2}{*}{$E M / S I A B$} \\
\hline & & & $\begin{array}{l}\text { Renda familiar } \\
\text { per capita }\end{array}$ & $\begin{array}{l}\text { Crianças } \\
<12 \text { anos (\%) }\end{array}$ & $\begin{array}{l}\text { Média de } \\
\text { escolaridade } \\
\text { entre 20-29 } \\
\text { anos }\end{array}$ & & & \\
\hline $60-2$ & 13,3 & 1,7 & 1,9 & 14,6 & 13,1 & 34,0 & 0,2 & 0,1 \\
\hline $60-3$ & 18,4 & 1,8 & 2,4 & 15,2 & 10,7 & 41,2 & 0,2 & 0,1 \\
\hline $60-4$ & 15,3 & 2,2 & 1,9 & 19,8 & 10,3 & 24,1 & 0,5 & 0,5 \\
\hline $60-5$ & 19,8 & 2,1 & 2,0 & 16,6 & 10,0 & 26,1 & 0,4 & 0,4 \\
\hline $60-6$ & 18,2 & 2,1 & 1,3 & 18,5 & 10,9 & 13,5 & 0,5 & 0,6 \\
\hline $61-1$ & 22,5 & 1,7 & 2,4 & 11,2 & 12,6 & 34,8 & 0,1 & 0,1 \\
\hline $61-3$ & 16,4 & 1,9 & 2,2 & 18,1 & 13,7 & 29,9 & 0,3 & 0,3 \\
\hline $61-4$ & 11,3 & 1,9 & 1,7 & 17,2 & 9,5 & 9,6 & 0,6 & 0,5 \\
\hline $61-5$ & 17,9 & 2,1 & 2,1 & 16,0 & 10,1 & 17,4 & 0,4 & 0,5 \\
\hline $61-6$ & 6,0 & 2,5 & 1,1 & 33,4 & 6,2 & 13,9 & 1,0 & 1,0 \\
\hline Barra Funda & 16,2 & 2,0 & 1,9 & 17,7 & 10,8 & 25,0 & - & - \\
\hline
\end{tabular}


áreas da Barra Funda obtêm os valores mais favoráveis, ficando a microárea "61-6" na 17ạ posição, com valores próximos aos valores globais do Butantã.

Em seguida, as microáreas foram ordenadas segundo seus valores de EMSV, em cada uma das áreas de estudo. As Tabelas 3 e 4 apresentam as microáreas ordenadas segundo o EMSV, em que fica evidenciada a proximidade geográfica das microáreas que receberam valores próximos de escore de situação de vida. Fundamentando-se neste procedimento de or-

Tabela 3

Escore médio de situação de vida (EMSV) segundo microárea, e respectiva subárea na favela da área do Butantã, São Paulo, Brasil.

\begin{tabular}{lll}
\hline Microárea & EMSV & Subárea \\
\hline $38-3$ & 0,06 & Alto \\
$37-2$ & 0,17 & Alto \\
$38-4$ & 0,20 & Alto \\
$37-6$ & 0,22 & Alto \\
$38-1$ & 0,31 & Alto \\
$38-6$ & 0,32 & Vila Clô \\
$37-4$ & 0,36 & Alto \\
$37-3$ & 0,60 & Riacho Doce \\
$38-2$ & 0,74 & Vila Clô \\
$37-5$ & 0,78 & Riacho Doce \\
$37-1$ & 0,90 & Riacho Doce \\
$38-5$ & 0,90 & Riacho Doce \\
\hline
\end{tabular}

Tabela 4

Escore médio de situação de vida (EMSV) segundo microárea, e respectiva subárea na área da Barra Funda, São Paulo, Brasil.

\begin{tabular}{lll}
\hline Microárea & EMSV & Subárea \\
\hline $61-1$ & 0,07 & Santa Cecília \\
$60-3$ & 0,17 & Condomínio \\
$60-2$ & 0,23 & Condomínio \\
$61-3$ & 0,26 & Santa Cecília \\
$60-5$ & 0,37 & Santa Cecília \\
$61-5$ & 0,42 & Bom Retiro \\
$60-4$ & 0,46 & Santa Cecília \\
$60-6$ & 0,53 & Santa Cecília \\
$61-4$ & 0,55 & Bom Retiro \\
$61-6$ & 0,98 & Favela Aldeínha \\
\hline
\end{tabular}

denação, foi possível definir três subáreas geograficamente contíguas dotadas de características aproximadamente homogêneas de seus indicadores de vida, na região do Butantã, e quatro subáreas na região da Barra Funda.

As microáreas que compõem as áreas de abrangência de PSF nas duas regiões foram agregadas consoante proximidade geográfica e semelhança de valor de EMSV, resultado de agregação de alguns indicadores obtidos pelo cadastro - tempo de moradia no bairro, renda familiar per capita, escolaridade, proporção de indivíduos menores de 12 anos, cobertura de convênio de saúde e número de indivíduos por cômodo. Estas microáreas com indicadores de vida semelhantes são, efetivamente, vizinhas entre si, compondo, assim, subáreas aproximadamente homogêneas.

Na região periférica (Butantã), foram identificadas três subáreas. A subárea do "Alto" é constituída por seis microáreas onde os indicadores de situação de vida se apresentaram com os valores mais favoráveis. Esta subárea possui uma infra-estrutura de arruamento, com diversas ruas asfaltadas, permitindo a circulação de veículos e coleta de lixo. $\mathrm{O}$ aspecto visual das moradias é relativamente melhor, e verifica-se a presença mais evidente de pequenos comércios acoplados à moradia.

A subárea do Riacho Doce é o núcleo mais antigo de ocupação da favela, e fica próximo às margens de um córrego fortemente poluído por esgoto e que apresenta freqüentes trasbordamentos em época de chuvas. $\mathrm{O}$ arruamento é bastante precário, impossibilitando a circulação de veículos em boa parte das ruas, situação que facilita o acúmulo de lixo.

A subárea da Vila Clô é o núcleo de invasão mais recente, ocupando parte de dois quarteirões onde já havia dezenas de casas em situação regular. Este núcleo possui habitações com aspecto bastante precário, e os seus moradores vivem sob a ameaça, ainda que virtual, de serem despejados (pelo fato de ser área de invasão muito recente). Não se verifica uma identidade de comunidade entre esta subárea e as duas primeiras.

Na região central (Barra Funda) foram identificadas quatro subáreas. A subárea da Favela Aldeinha, com os piores desempenhos do EMSV na região da Barra Funda e cujos indicadores apresentam valores mais próximos aos encontrados no Butantã (área de favela) é um local de invasão relativamente recente, com péssimas condições de urbanização e habitação, em que prevalecem moradias construídas com materiais reaproveitados.

A subárea do Bom Retiro é a que possui forte presença de cortiços e migrantes clandesti- 
nos (bolivianos) que trabalham nas inúmeras pequenas confecções têxteis aí instaladas. Vale destacar que esta população, sujeita a precárias condições de vida, acaba por ter, ainda, grande dificuldade de acesso aos recursos sociais disponíveis, decorrente da situação de migração não regularizada.

A subárea de Santa Cecília é composta por uma população de melhor renda e escolaridade, possuindo como característica marcante o predomínio de indivíduos idosos e já aposentados, antigos moradores do bairro.

A quarta subárea (Condomínio), constituída predominantemente por condomínios fechados, possui população mais jovem, e destaca-se pelo padrão de habitação e de rendimento familiar superiores.

Note-se a diferença importante entre as áreas periférica e central: a segunda exibe índices marcadamente mais favoráveis dos indicadores de situação de vida, sobretudo os referentes à renda per capita, à escolaridade, à média de pessoas por cômodo utilizado para dormir e à cobertura de convênio.

\section{Escore médio de situação de vida (EMSV) e opinião dos agentes comunitários de saúde (ACS)}

Esta análise, realizada apenas para a área do Butantã, mostra uma evidente correlação entre cada um dos seis indicadores de situação de vida e os escores de opinião dos ACS, para o conjunto das 12 microáreas, como pode ser observado na Tabela 1.

Existe uma correlação linear significativa entre o EMSV de cada microárea e o escore de opinião dos ACS, com $\mathrm{R}^{2}=0,82(0,48<\mathrm{R}<0,95)$, $\beta=0,737$ (IC: $0,423-1,051$ ). Ou seja, os indicadores de situação de vida agregados em um escore são coincidentes com a impressão mais subjetiva que os ACS construíram acerca das 12 microáreas, quando as hierarquizaram segundo a impressão de serem mais ou menos problemáticas.

\section{Escore médio de situação de vida (EMSV) e escore médio do SIAB (EM/SIAB)}

A comparação entre os valores do EMSV e o escore médio que incluiu apenas as variáveis disponíveis na Ficha A do SIAB (EM/SIAB) mostrou um coeficiente de correlação linear muito alto, nas duas áreas estudadas.

No Butantã, a correlação apresentou $\mathrm{R}^{2}=$ 0,98 , com intervalo de confiança de $\mathrm{R}$ entre 0,89 e 0,99 , e $\beta=0,875$ (IC: 0,775-0,976). A correlação linear entre o EM/SIAB e o escore de opi- nião dos ACS apresenta $\mathrm{R}^{2}=0,64$, com intervalo de confiança de R entre 0,41 e 0,94, e $\beta=0,802$ (IC: 0,427-1,177); portanto, sem diferença significativa com o valor de $\mathrm{R}^{2}$ encontrado na correlação entre o EMSV e a opinião dos ACS.

Na Barra Funda, a correlação entre o EMSV e o EM/SIAB apresentou $\mathrm{R}^{2}=0,97$, com intervalo de confiança de $R$ entre 0,89 e 0,99 , e $\beta=0,947$ (IC: 0,796-1,098).

\section{Escore médio de situação de vida (EMSV) e agravos referidos}

Na área do Butantã, não foi encontrada correlação significativa entre o EMSV de cada microárea com os coeficientes de prevalência de cada agravo referido.

$\mathrm{Na}$ área da Barra Funda, foi encontrada correlação significativa apenas entre o EMSV e o diabetes, que apresenta maior prevalência nas microáreas com menor EMSV (indicador mais favorável), com $\mathrm{R}^{2}=0,48$ e $\beta=-0,132$ (IC: $-0,227$ $-0,037)$.

Observa-se uma grande variação da prevalência dos agravos referidos entre as diversas microáreas. Na Barra Funda, a hipertensão, o diabetes, o tabagismo e a bronquite foram os agravos com menor variação na prevalência, com proporções entre maior e menor prevalência de 1,$7 ; 2,9 ; 2,4$ e 2,2, respectivamente. No Butantã, onde estes também foram os agravos com menor variabilidade na prevalência, os valores ficaram em 1,8; 3,6; 2,9 e 3,6, respectivamente.

\section{Discussão}

A coleta de dados pessoais e familiares que possam ser utilizados como indicadores de situação de vida permite que o cadastro seja capaz de identificar subáreas de população exposta a piores condições de vida. Permite, desse modo, identificar os locais onde a iniqüidade sóciocultural e econômica deve expor mais intensamente os indivíduos a agravos à saúde, passo fundamental para um planejamento de ações em saúde, seja na unidade de saúde, seja na comunidade, que possa dar respostas apropriadas para esta diversidade de situações de vida e saúde.

A utilização de indicador composto por escores médios de indicadores primários permite instrumentalizar adequadamente os processos de gerenciamento local das ações em saúde, na medida em que podem identificar e discriminar subáreas com necessidades em saúde diferenciadas. 
A evidente colinearidade entre alguns dos indicadores de vida que compuseram o EMSV abre a possibilidade de utilização de um número menor de indicadores para a construção de um indicador composto.

A forte correlação entre o EMSV e o EM/SI$\mathrm{AB}$ leva à conclusão de que os dados considerados pelo SIAB, colhidos por meio da Ficha $\mathrm{A}$ seriam suficientes para caracterizar diferenças e iniqüidade presentes em uma área adscrita por uma unidade de saúde. Porém, esta conclusão deve ainda ser considerada com maior cuidado, pois o estudo foi realizado apenas em duas áreas de um município de grande porte como São Paulo.

Considerando as dificuldades operacionais enfrentadas pelo PSF em relação ao sistema de informações, em que se destacam dificuldades na coleta dos dados, na consolidação na unidade de saúde e sobretudo em sua análise em âmbito local (http://dtr2001.saude.gov.br/psf/ /publicacoes/download/relpsf2.zip, acessado em 09/Nov/2003) é inteiramente desejável que não se amplie o universo de dados colhidos pelo cadastramento das famílias; todavia, parece também inteiramente desejável que o sistema

\section{Resumo}

Com a implantação do Programa de Saúde da Família no Município de São Paulo, Brasil, foi realizada experiência piloto de um cadastro ampliado para coleta de informações sobre famílias em dois centros de saúde escola. O objetivo do estudo é a análise deste cadastro como instrumento gerencial que possa discriminar diferenças e iniqüidades presentes em cada área adstrita. Com base em dados coletados, foram construídos seis indicadores relativos a tempo de moradia, número de pessoas por cômodo para dormir, renda familiar per capita, percentagem de crianças, escolaridade e cobertura de convênio de saúde. Com estes seis indicadores foi elaborado um indicador composto, o Escore Médio de Situação de Vida (EMSV), bem como o Escore Médio/SIAB (EM/SIAB) com os três indicadores que estão disponíveis na Ficha A do SIAB. O resultado identificou subáreas geograficamente contíguas com situações de vida distintas. O EM/SIAB apresentou poder discriminatório muito próximo ao EMSV. Concluise pela possibilidade de diferenciar e discriminar subáreas, apontando para a necessidade de organizar distintas ações em saúde para cada subárea.

Programa Saúde da Família; Sistemas de Informação; Gerência informatizado possa emitir relatórios que permitam uma fácil visualização da diversidade microrregional, dado que uma parcela significativa das unidades de saúde não dispõe de profissionais responsáveis pela gerência capacitados para a manipulação de dados brutos para a elaboração de relatórios sintéticos que permitam tal visualização.

A ausência de correlação entre a prevalência de agravos e condições referidas de saúde pode estar revelando tão somente a dificuldade de obtenção de dados precisos e confiáveis sobre o adoecimento das pessoas, colhido no domicílio por um agente comunitário de saúde. Reforça esta hipótese o fato de que os coeficientes de agravos variam muito de um ACS para outro, mesmo quando consideradas microáreas com indicadores de situação de vida semelhantes. O desempenho individual do ACS e a sua facilidade em estabelecer relação amigável e de confiança com o morador que está sendo cadastrado podem estar determinando o resultado final deste perfil, o que leva à conclusão de que a utilização destes dados com o intuito de traçar perfis de adoecimento deve ser avaliada com bastante cautela.

\section{Colaboradores}

A. Sala, O. Simões e C. G. Luppi participaram do planejamento da pesquisa, desenho metodológico, análise dos dados e elaboração final do artigo. A. Sala contribuiu na implementação do trabalho de campo no Centro de Saúde Escola Prof. Samuel B. Pessoa e na redação final do texto. M. C. Mazziero colaborou na coordenação da fase de campo da pesquisa no Centro de Saúde Escola da Barra Funda.

\section{Agradecimentos}

À Professora Hillegonda Maria Dutilh Novaes, do Departamento de Medicina Preventiva da Faculdade de Medicina da Universidade de São Paulo, por suas críticas e sugestões. Às equipes do Centro de Saúde Escola Barra Funda - Alexandre Vranjac e Centro de Saúde Escola Prof. Samuel B. Pessoa, pelo apoio logístico e operacional. 


\section{Referências}

1. Sala A. O registro médico em serviços ambulatoriais: a anotação como componente da técnica em saúde [Tese de Doutorado]. São Paulo: Faculdade de Medicina, Universidade de São Paulo; 1998.

2. Ministério da Saúde. Programa de Saúde da Família. Brasília: Secretaria Executiva, Ministério da Saúde; 2001.

3. Viana ALD, Dal Poz MR. A reforma do sistema de saúde no Brasil e o Programa de Saúde da Família. Physis 1998; 8:11-48.

4. Santana ML, Carmagnani, MI. Programa saúde da família no Brasil: um enfoque sobre seus pressupostos básicos, operacionalização e vantagens. Saúde Soc 2001; 10:33-53.

5. Trad LAB, Bastos ACS. O impacto sócio-cultural do Programa de Saúde da Família (PSF): uma proposta de avaliação. Cad Saúde Pública 1998; 14: 429-35.

6. Paim JS. Abordagens teórico-conceituais em estudos de condições de vida e saúde: notas para reflexão e ação. In: Barata RB, organizador. Condições de vida e situação de saúde. Rio de Janeiro: ABRASCO; 1997. p. 7-30.
7. Mendes EV, Teixeira CF, Araújo EC, Cardoso MRL. Distritos sanitários: conceitos-chave. In: Mendes EV, organizador. Distrito sanitário: o processo social de mudança das práticas sanitárias do Sistema Único de Saúde. 3a Ed. São Paulo: Editora Hucitec/Rio de Janeiro: ABRASCO; 1995. p. 159-85.

8. Akerman M. Metodologia de construção de indicadores compostos: um exercício de negociação intersetorial. In: Barata RB, organizador. Condições de vida e situação de saúde. Rio de Janeiro: ABRASCO; 1997. p. 95-113.

9. Barros MB. Epidemiologia e superação das iniqüidades em saúde. In: Barata RB, Barreto ML, Almeida Filho N, Veras RP, organizadores. Eqüidade e saúde: contribuições da epidemiologia. Rio de Janeiro: Editora Fiocruz/ABRASCO; 1997. p. 161-76.

Recebido em 11/Dez/2003

Versão final reapresentada em 26/Mai/2004

Aprovado em 31/Mai/2004 